\title{
Fe-Centers in GaN as Candidates for Spintronics Applications
}

Enno Malguth, Axel Hoffmann ${ }^{1}$, Matthew Phillips, and Wolfgang Gehlhoff ${ }^{1}$

Microstructural Analysis Unit, University of Technology, Sydney, NSW 2007, Australia

${ }^{1}$ Technische Universität Berlin, Institut für Festkörperphysik, 10623 Berlin, Germany

\section{ABSTRACT}

The potential use of Fe doped GaN for spintronics applications requires a complete understanding of the electronic structure of $\mathrm{Fe}$ in all of its charge states. To address these issues, a set of $400 \mu \mathrm{m}$ thick freestanding HVPE grown GaN:Fe crystals with different Fe-concentration levels ranging from $5 \times 10^{17} \mathrm{~cm}^{-3}$ to $2 \times 10^{20} \mathrm{~cm}^{-3}$ was studied by means of photoluminescence, photoluminescence excitation (PLE) and Fourier transform infrared (FTIR) transmission experiments. The $\mathrm{Fe}^{3+/ 2+}$ charge transfer (CT) level was determined to be at $2.86 \pm 0.01 \mathrm{eV}$ above the valence band maximum considerably lower than the previously reported value of $3.17 \pm 0.10 \mathrm{eV}$. A bound state of the form $\left(\mathrm{Fe}^{2+}, \mathrm{h}_{V B}\right)$ with a binding energy of $50 \pm 10 \mathrm{meV}$ has been established as an excited state of $\mathrm{Fe}^{3+}$. FTIR transmission measurements revealed an internal $\left({ }^{5} \mathrm{E}-{ }^{5} \mathrm{~T}_{2}\right)$ transition of $\mathrm{Fe}^{2+}$ around $400 \mathrm{eV}$ which, until now, was believed to be degenerate with the conduction band. Consequently, a second CT band was detected in PLE spectra.

\section{INTRODUCTION}

A possible realization of spintronic devices is to introduce transition metal (TM) ions into a wide band gap semiconductor [1]. Theoretically, in such a diluted magnetic semiconductor (DMS) the magnetic order of the localized magnetic centers is established by delocalized free carriers, resulting in room temperature ferromagnetism [2, 3]. In particular, free holes are shown to interact with the magnetic impurities via exchange interaction [3]. Therefore, in order to predict the feasibility of carrier-mediated spin coupling, it is crucial to know the electronic structure of the TM centers, which depends on the present charge state and is strongly influenced by the host crystal [4]. The exact position of the $\mathrm{Fe}^{3+/ 2+}$ charge transfer $(\mathrm{CT})$ level within the bandgap is of particular interest. While extensive studies have been conducted on the promising GaN:Mn material system, e.g. [5], only little is known about the applicability of GaN:Fe for spintronics.

The spinforbidden $\left({ }^{4} \mathrm{~T}_{1}(\mathrm{G})-{ }^{6} \mathrm{~A}_{1}(\mathrm{~S})\right)$ transition of the $\mathrm{Fe}^{3+}$ center in GaN exhibits a structured near infra red (IR) luminescence at $1.299 \mathrm{eV}$ with a $8 \mathrm{~ms}$ lifetime [6,7]. By means of photoluminescence excitation (PLE) Heitz et al. identified higher excited states, ${ }^{4} \mathrm{~T}_{2}(\mathrm{G})$ and ${ }^{4} \mathrm{E}(\mathrm{G})$, at $2.01 \mathrm{eV}$ and $2.731 \mathrm{eV}$, respectively, as well as a shallow bound state of the form $\left(\mathrm{Fe}^{3+}, \mathrm{e}, \mathrm{h}\right)$ at $2.888 \mathrm{eV}$ [8]. After pinpointing the $\mathrm{Fe}^{3+/ 2+} \mathrm{CT}$ level at $(3.17 \pm 0.10) \mathrm{eV}$, the authors drew three major conclusions [8]: (1) The binding energy of the bound state equals $\approx 280 \mathrm{meV}$. (2) With a likely energy of approximately $390 \mathrm{meV}$ the $\mathrm{Fe}^{2+}\left({ }^{5} \mathrm{E}-{ }^{5} \mathrm{~T}_{2}\right)$ transition is degenerate with the conduction band and cannot be observed. (3) The internal reference rule fails for GaN in conjunction with both GaAs and AlN [9, 10]. 


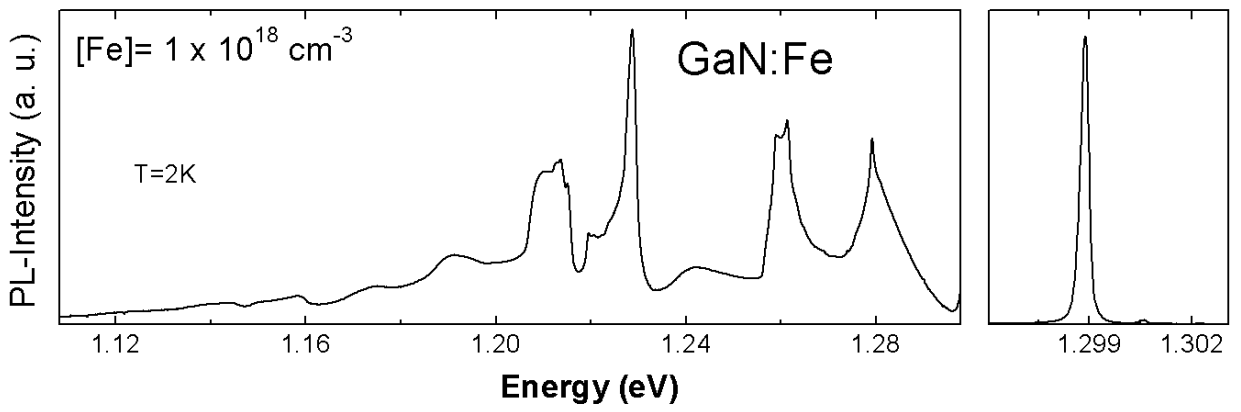

Figure 1. Low-temperature $(2 \mathrm{~K})$ luminescence of the $\mathrm{Fe}^{3+}\left({ }^{4} \mathrm{~T}_{1}(\mathrm{G})-{ }^{6} \mathrm{~A}_{1}(\mathrm{~S})\right)$ transition in GaN for a Fe concentrations of $1 \times 10^{18} \mathrm{~cm}^{-3}$. The dominating ZPL at $1.299 \mathrm{eV}$ is displayed at a different energy scale for better resolution.

In this work we present a number of optical studies on a set of high quality GaN samples doped with iron at varying concentrations. Both charge states $3+$ and $2+$ are observed. Their respective electronic structure is studied in detail as well as $\mathrm{Fe}^{3+/ 2+} \mathrm{CT}$ processes. Previous results on the $\mathrm{Fe}^{3+}$ center are determined more precisely and partly revised. The concentration dependency of the presented results provides an indication of the ideal Fe concentration for the potential realization of a GaN:Fe based DMS.

\section{EXPERIMENTAL DETAILS}

The samples of hexagonal crystal phase were grown along the c-axis by ATMI using HVPE. Fe was incorporated during the growth process at concentrations ranging from $5 \times 10^{17} \mathrm{~cm}^{-3}$ to $2 \times 10^{20} \mathrm{~cm}^{-3}$. The substrate was removed from the $\sim 500 \mu \mathrm{m}$ thick layers resulting in freestanding samples. Photoluminescence (PL) experiments were carried out with the samples immersed in liquid helium exciting with an Ar-ion laser at $2.41 \mathrm{eV}$. The PL was dispersed by a $1 \mathrm{~m}$ Spex double monochromator and detected by a liquid nitrogen cooled germanium-detector. In photoluminescence excitation (PLE) experiments a tungsten-halogen lamp in combination with a $275 \mathrm{~mm}$ double monochromator served as tunable light source. The luminescence was dispersed and detected by a $300 \mathrm{~mm}$ double monochromator and a germanium-detector, respectively. Fourier transform infrared (FTIR) measurements were perfomed using a Bruker IFS 66v commercial Fourier spectrometer fitted with a liquid helium cryostat. For PL and FTIR experiments the samples were mounted in such a way that light travelled perpendicular to the c-axis, resulting in an absorption path length equal to the samples' widths ( $\sim 2 \mathrm{~mm})$, which produced increased absorption. This method also enabled variations in the incoming light's polarization with respect to the c-axis.

\section{RESULTS AND DISCUSSION}

Figure 1 presents PL spectra of the internal $\mathrm{Fe}^{3+}\left({ }^{4} \mathrm{~T}_{1}(\mathrm{G})-{ }^{6} \mathrm{~A}_{1}(\mathrm{~S})\right)$ transition in GaN at $1.299 \mathrm{meV}$ and its phonon sideband for different Fe concentrations [6]. Both ZPL and 


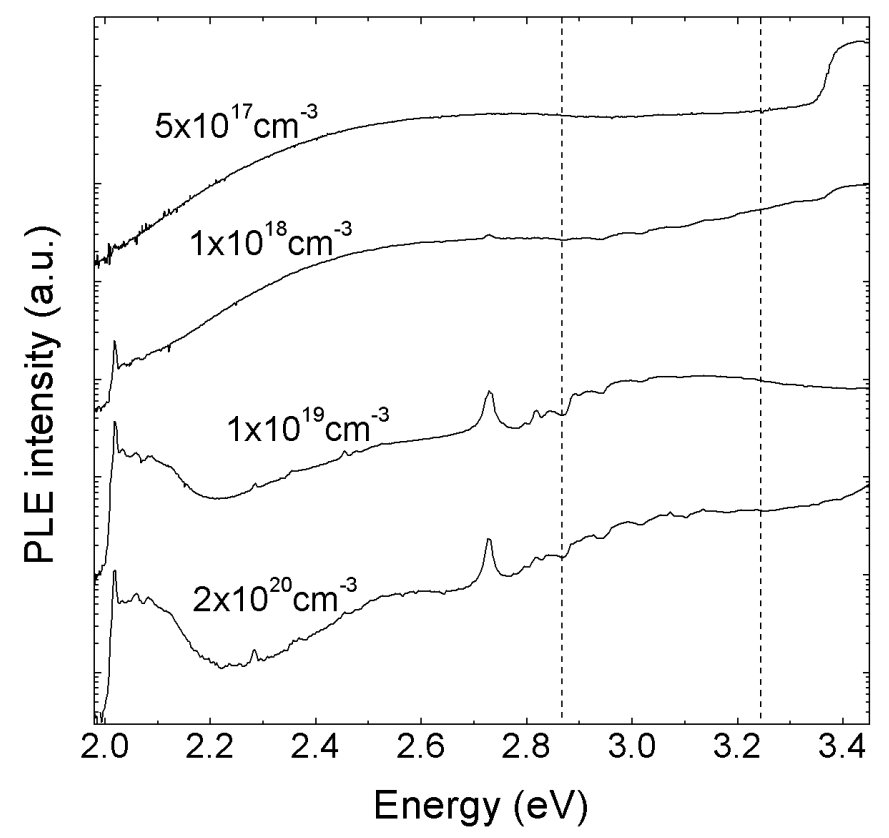

Figure 2. PLE spectra of the $\mathrm{Fe}^{3+}\left({ }^{4} \mathrm{~T}_{1}(\mathrm{G})-{ }^{6} \mathrm{~A}_{1}(\mathrm{~S})\right)$ luminescence at 1.299 eV of Fedoped GaN for various Fe concentrations (given in $\mathrm{cm}^{-3}$ ) at $7 \mathrm{~K}$ on logarithmic scale. The luminescence was detected in an $8 \mathrm{meV}$ wide window around $1.299 \mathrm{eV}$.

sideband are similar for any studied concentration (not shown here). The full width at half maximum (FWHM) of the ZPL scales with the Fe concentration between 120 and $270 \mu \mathrm{eV}$, proving the samples to be of better quality than those studied previously [7]. This is mainly regarding the strain free incorporation of Fe on Ga site and was confirmed by electron paramagnetic resonance (EPR) experiments.

The most pronounced features of the sideband could successfully be assigned to phonon modes of the host crystal and local vibrational modes (LVM) [11-14]. A strong coupling of the $\mathrm{Fe}^{3+}$ center on only $\mathrm{E}_{2}$ phonon modes as well as on A1 and E LVMs was identified.

Figure 2 shows PLE spectra of the $\mathrm{Fe}^{3+}\left({ }^{4} \mathrm{~T}_{1}(\mathrm{G})-{ }^{6} \mathrm{~A}_{1}(\mathrm{~S})\right)$ luminescence in GaN for different Fe concentrations. Transitions into the higher excited $\mathrm{Fe}^{3+}$ states, ${ }^{4} \mathrm{~T}_{2}(\mathrm{G})$ and ${ }^{4} \mathrm{E}(\mathrm{G})$, are reflected by peaks at $2.01 \mathrm{eV}$ and $2.73 \mathrm{eV}$ respectively which appear at $\mathrm{Fe}$ concentrations from $1 \times 10^{18} \mathrm{~cm}^{-3}$. A smaller peak at $2.812 \mathrm{eV}$ with pronounced replicas was previously attributed by Heitz et al. to an exciton bound to the $\mathrm{Fe}^{3+}$ center $\left(\mathrm{Fe}^{3+}, \mathrm{e}, \mathrm{h}\right)[8]$. However, in Ref. [8] the first phonon replica at $2.888 \mathrm{eV}$ was mistaken for the actual ZPL. The observed strong phonon coupling is typical for bound states [8] and can be explained by a coupling on the $\mathrm{E}_{2}$ phonon mode and the $\mathrm{A}_{1} \mathrm{LVM}$. The replicas are superimposed on the start of a broad UV band which represents the CT process

$$
F e^{3+}\left({ }^{6} A_{1}(S)\right)+h \nu \longrightarrow F e^{2+}+h_{V B}
$$

in which a hole is excited into the valence band [8]. Therefore, the band's low energy onset equals the distance between the $\mathrm{Fe}^{3+/ 2+} \mathrm{CT}$ level and the VB maximum. An exponential 
fit produces an energy of $(2.865 \pm 0.01) \mathrm{eV}$. This is considerably lower than the value of $(3.17 \pm 0.10) \mathrm{eV}$ established by Heitz et al. on the basis of the questionable energy found for the bound state [8]. As a result the binding energy of the bound state amounts to only $(50 \pm 10) \mathrm{meV}$, indicating a small overlap of hole and core state wave functions. With the CT level less close to the CB minimum, there will only be a minor hybridization of the Fe-center's core states with the CB. Both points imply only a weak exchange interaction. Therefore, in line with the Fe center in other III-V materials [15-17], the bound state is better described as an effective-mass-like complex consisting of a hole bound to a $\mathrm{Fe}^{2+}$ center, $\left(\mathrm{Fe}^{2+}, \mathrm{h}_{V B}\right)$.

An additional consequence of the new location of the $\mathrm{Fe}^{3+/ 2+} \mathrm{CT}$ level, $(0.68 \pm$ $0.06) \mathrm{eV}$ below the conduction band minimum, is that the $\mathrm{Fe}^{2+}\left({ }^{5} \mathrm{E}-{ }^{5} \mathrm{~T}_{2}\right)$ transition might be located within the band gap.

According to the PLE spectra (figure 2), the CT process (Eq. 1) initiates the most efficient excitation process of the $\mathrm{Fe}^{3+}$ center, i.e. the capture of a free hole by an $\mathrm{Fe}^{2+}$ center, probably by forming the discussed bound state, which subsequently relaxes nonradiatively into the ${ }^{4} \mathrm{~T}_{1}(\mathrm{G})$ state.

$$
F e^{2+}+h_{V B} \longrightarrow\left(F e^{2+}, h\right) \stackrel{\text { nonrad. }}{ }>F^{3+}\left({ }^{4} T_{1}\right)
$$

A second band resembling a CT process can be seen particularly for $[\mathrm{Fe}]=2 \times 10^{20} \mathrm{~cm}^{-3}$ (figure 2) starting at $(3.245 \pm 0.02) \mathrm{eV}$. Its origin can be tentatively identified as the CT resulting in the excited $\mathrm{Fe}^{2+}$ state, ${ }^{4} \mathrm{~T}_{2}(\mathrm{G})$. This assumption is supported by the observation of the $\left({ }^{5} \mathrm{E}-{ }^{5} \mathrm{~T}_{2}\right)$ transition of $\mathrm{Fe}^{2+}$ presented later on in this work. The two $\mathrm{Fe}^{2+}$ states will be shown to be separated by $386 \mathrm{meV}$ which perfectly agrees with the energetic difference of $(380 \pm 20) \mathrm{meV}$ between the onsets of the alleged CT bands observed here.

The intensity of the $\mathrm{Fe}^{3+}$ related characteristics of the PLE spectra in figure 2 suggest that at Fe concentrations of $1 \times 10^{18} \mathrm{~cm}^{-3}$ and $5 \times 10^{17} \mathrm{~cm}^{-3}$ there are fewer and no ions in the $3+$ charge state, respectively. At these concentrations the $\mathrm{Fe}^{3+}$ luminescence is primarily excited via an external generation of free holes recombining with $\mathrm{Fe}^{2+}$ centers (equation 2). The origin of free holes is probably a two step process involving the deep defect centers responsible for the yellow luminescence [18]. This assumption is also supported by the fact that a wide excitation band starting at $2.1 \mathrm{eV}$ appears in both PLE spectra of the yellow luminescence [18] and of the $\mathrm{Fe}^{3+}$ emission (figure 2). Considering that the $\mathrm{Fe}^{3+}$ luminescence in figure 1 was excited at $2.41 \mathrm{eV}$, here the hole transfer to $\mathrm{Fe}^{3+}$ centers represents the dominating excitation process at any studied Fe concentration.

FTIR transmission spectra of the Fe-doped GaN samples are displayed in figure 3. A richly structured absorption band around $395 \mathrm{meV}$ can be observed for any concentration. It can be unambiguously assigned to the $\left({ }^{5} \mathrm{E}-{ }^{5} \mathrm{~T}_{2}\right)$ transition of $\mathrm{Fe}^{2+}$ based on three arguments: (i) The observed absorption structure is found in the same energy region and is of similar shape as the $\mathrm{Fe}^{2+}$ transition in GaAs, GaP and $\mathrm{InP}$, i.e. between 340 and $450 \mathrm{meV}$ [19]. (ii) The shape and amplitude of the absorption feature change significantly with the Fe concentration. (iii) Its position equals the distance between the two CT bands yielded by PLE measurements. Consequently, the spectra in figure 3 confirm the presence of iron in the $2+$ charge state at any Fe concentration studied, as was concluded on the 


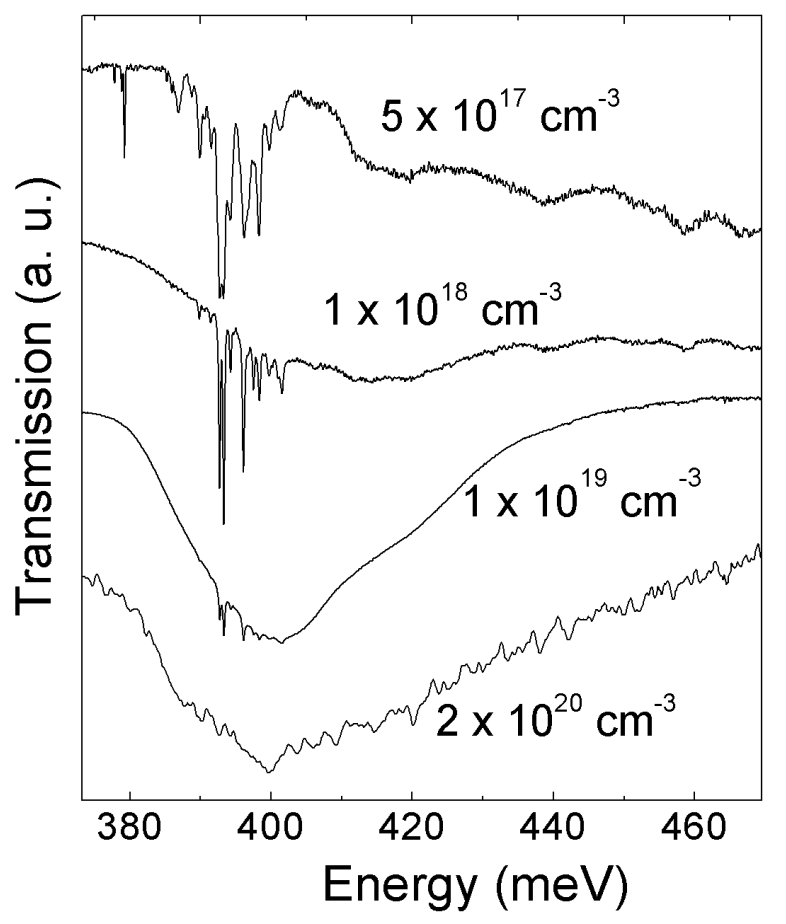

Figure 3. FTIR transmission spectra of the $\left({ }^{5} \mathrm{E}-{ }^{5} \mathrm{~T}_{2}\right)$ transition of $\mathrm{Fe}^{2+}$ in $\mathrm{GaN}$ for different Fe concentrations. The noisy signal for $[\mathrm{Fe}]=2 \times 10^{20} \mathrm{~cm}^{-3}$ is the result of the very high overall absorption of this sample.

basis of PLE experiments. $\mathrm{Fe}^{2+}$ is a $\mathrm{d}^{6}$ system with a ${ }^{5} \mathrm{D}$ ground state. In compound semiconductor crystals of zinc blende structure, the ground state splits under the impact of the crystal field of $\mathrm{T}_{d}$ symmetry into the ground state, ${ }^{5} \mathrm{E}$, and the excited state, ${ }^{5} \mathrm{~T}_{2}$. Spin-orbit-interaction causes these states to split into 5 and 6 sublevels, respectively. The resulting electronic structure has been established successfully for GaAs, GaP and InP [19]. However, in hexagonal crystals of wurtzit structure the crystal field is of $\mathrm{c}_{3 V}$ symmetry causing an additional splitting which has not been resolved so far and which is reflected by the complex absorption structure appearing in figure 3. According to Boltzmann's law, the elevated temperatures encountered during the experiments caused most of the ground states to be populated. This produced a multitude of absorption lines which are difficult to assign.

It can be seen from figure 3 that an increase in Fe concentration produces a broad absorption band at the position of the Fe2+ absorption around $400 \mathrm{meV}$, superimposing the finely structured absorption spectrum. The mechanisms behind this are most likely inter-center interaction and localized strain, which occur at high $\mathrm{Fe}$ densities and cause the single $\left({ }^{5} \mathrm{E}-{ }^{5} \mathrm{~T}_{2}\right)$ lines to broaden.

\section{CONCLUSION}

A consistent picture of iron in GaN has been established in this work. Both charge states $3+$ and $2+$ were detected for different Fe concentrations. The respective electronic 
structure was established. Significant findings relevant to potential spintronics application include: the position of the CT level (2.865 eV above the VB), the existence of the bound state, $\left(\mathrm{Fe}^{2+}, \mathrm{h}\right)$, constituting a transient shallow acceptor state, and the confirmation that the $\mathrm{Fe}^{2+}\left({ }^{5} \mathrm{E}-{ }^{5} \mathrm{~T}_{2}\right)$ transition is located within the band gap. These results will hopefully stimulate further research activities towards carrier-mediated ferromagnetism in GaN:Fe.

\section{References}

[1] S. J. Pearton, C. R. Abernathy, G. T. Thaler, R. M. Frazier, D. P. Norton, F. Ren, Y. D. Park, J. M. Zavada, I. A. Buyanova, W. M. Chen and A. F. Hebard, Journal of Physics: Condensed Matter page R209 (2004).

[2] H. Ohno, Science 281, 951 (1998).

[3] T. Dietl, H. Ohno and F. Matsukura, Physical Review B 63, 195205(2001).

[4] F. S. Ham and G. A. Slack, Physical Review B 4, 777 (1971).

[5] T. Dietl, H. Ohno, F. Matsukura, J. Cibert and D. Ferrand, Science 287, 1019 (2000).

[6] K. Maier, M. Kunzer, U. Kaufmann, J. Schneider, B. Monemar, I.Akasaki and H. Amano, Materials Science Forum 143-147, 93 (1994).

[7] R. Heitz, P. Thurian, I. Loa, L. Eckey, A. Hoffmann, I. Broser, K. Pressel, B. K. Meyer and E. N. Mokhov, Applied Physics Letters 67, 2822 (1995).

[8] R. Heitz, P. Maxim, L. Eckey, P. Thurian, A. Hoffmann, I. Broser, K. Pressel and B. K. Meyer, Physical Review B 55, 4382 (1997).

[9] J. M. Langer and H. Heinrich, Phys. Rev. Lett. 55, 1414 (1985).

[10] M. L. J. M. Langer, C. Delerue and H. Heinrich, Physical Review B 38, 7723 (1988).

[11] H. Siegle, L. Eckey, A. Hoffmann, C. Thomsen, B. K. Meyer, D. Schikora, M. Hankeln and K. Lischka, Solid State Communications 96, 943 (1995).

[12] V. Y. Davydov, Y. E. Kitaev, I. N. Goncharuk, A. N. Smirnov, J. Graul, O. Semchinova, D. Uffmann, M. B. Smirnov, A. P. Mirgorodsky and R. A. Evarestov, Physical Review B 58, 12899 (1998).

[13] P. Thurian, G. Kaczmarczyk, H. Siegle, R. Heitz, A. Hoffmann, I. Broser, B. K. Meyer, R. Hoffbauer and U. Scherz, Materials Science Forum 196-201, 1571 (1995).

[14] C. Goebel, C. Schrepel, U. Scherz, P. Thurian, G. Kaczmarczyk and A. Hoffmann, Materials Science Forum 258-263, 1173 (1997).

[15] L. Podlowski, R. Heitz, T. Wolf, A. Hoffmann, D. Bimberg, I. Broser and W. Ulrici, Materials Science Forum 143-147, 311 (1994).

[16] K. Pressel, G. Rückert, A. Dörnen and K. Thonke, Phys. Rev. B 46, 13171 (1992).

[17] K. Pressel, A. Dörnen, G. Rückert and K. Thonke, Phys. Rev. B 47, 16267 (1993).

[18] A. Hoffmann, L. Eckey, P. Maxim, J.-C. Holst, R. Heitz, D. M. Hofmann, D. Kovalev, G. Steude, D. Volm, B. K. Meyer, T. Detchprohm, H. Amano and I. Akasaki, Solid State Electronics 41, 275 (1997).

[19] L. Podlowski, R. Heitz, P. Thurian, A. Hoffmann and I. Broser, Journal of Luminescence 48, 252 (1994). 